\title{
Application of the new at-column dilution (ACD) modulator for the two-dimensional RPXHILIC analysis of Buddleja davidii
}

\author{
Yingzhuang Chen ${ }^{1,2} \cdot$ Lidia Montero ${ }^{1,3} \cdot$ Jiang Luo ${ }^{1,3} \cdot$ Junjie Li ${ }^{1,3} \cdot$ Oliver J. Schmitz ${ }^{1,3}$
}

Received: 25 September 2019 / Revised: 4 December 2019 / Accepted: 7 January 2020 / Published online: 21 January 2020

(C) The Author(s) 2020

\begin{abstract}
The focus of this study was the analysis of the complex chemical composition from different parts of Buddleja davidii, whose species are commonly known as ornamental plants and herbal medicines in many countries. As an herbal medicine, it has been utilized for stroke treatments, headache, wound healing, neurological disorder, etc. However, the understanding of its chemical matrices is still insufficient. Therefore, an online two-dimensional reversed phase liquid chromatography $\mathrm{x}$ hydrophilic interaction liquid chromatography (RPLCxHILIC) system coupled with mass spectrometry was applied for further detailed investigation of the chemical constituents in Buddleja dividii. In this two-dimensional liquid chromatography (2D-LC) method, a new atcolumn dilution (ACD) modulator was introduced in the 2D-LC system to solve the incompatibility problem of the mobile phase between two dimensions, which resulted in a 2D-LC analysis with high orthogonality. For the root extract, as one of the analyzed samples, the optimization of the ${ }^{1} \mathrm{D}$ and ${ }^{2} \mathrm{D}$ gradients was carried out carefully. With this new modulator, much better peak separation and better peak shape were achieved compared to two-dimensional liquid chromatography system using a traditional standard (TS) modulator. With a similar approach, the other four parts of Buddleja davidii were well separated. Comparing the different analyzed parts, flowers and leaves showed the most complex profiles. MS and MS/MS data were obtained successfully, which demonstrated the potential of the proposed RPLCxHILIC-MS system in the constituents' analysis of herbal medicine. However, due to the lack of reported reference information, 24 compounds could be tentatively identified.
\end{abstract}

Keywords RPLCxHILIC $\cdot$ At-column dilution $(\mathrm{ACD}) \cdot$ Herbal medicine $\cdot$ 2D-LC $\cdot$ Buddleja davidii

\section{Introduction}

ABC Highlights: authored by Rising Stars and Top Experts.

Electronic supplementary material The online version of this article (https://doi.org/10.1007/s00216-020-02392-3) contains supplementary material, which is available to authorized users.

Oliver J. Schmitz

oliver.schmitz@uni-due.de

1 Applied Analytical Chemistry, University of Duisburg-Essen, Universitaetsstr. 5, 45141 Essen, Germany

2 Key Laboratory of Phytochemical R\&D of Hunan Province, Key Laboratory of Chemical Biology \& Traditional Chinese Medicine Research, Ministry of Education, Hunan Normal University, Changsha 410081, Hunan, China

3 Teaching and Research Center for Separation (TRC), University of Duisburg-Essen, Universitaetsstr. 5, 45141 Essen, Germany
In recent decades, as a rising star in the field of chromatography, two-dimensional liquid chromatography (2D-LC) has attracted more and more attention by rapid developments in research of complex systems, such as natural products, environmental pollutants, food and pharmacy, proteomics, and metabolomics [1]. The largest benefit of 2D-LC is the dramatic increase in peak capacity that may be achieved, which is reflected in the component overlapping reduction [2].

In online comprehensive 2D-LC (LCxLC), where the sample is completely and continuously transferred from the first dimension $\left({ }^{1} \mathrm{D}\right)$ to the second dimension $\left({ }^{2} \mathrm{D}\right)$, the combination of orthogonal dimensions is usually hard to achieve because incompatible mobile phases are often used in both dimensions. For example, one of the most orthogonal combinations that can be used in 2D-LC is the combination of RPLC and HILIC because these two separation mechanisms present completely different selectivity for the retention of the 
analytes. Besides, the mobile phases used in RPLC and HILIC are miscible and compatible with detectors such as MS. Therefore, a 2D-LC system based on the coupling of RPLC and HILIC (HILICxRPLC or RPLCxHILIC) should theoretically be a promising system [1]. However, the compatibility of the mobile phases in such two dimensions is always a problematic and an important issue. The main reason is that the weak solvent in the ${ }^{1} \mathrm{D}$ consists of a strong eluent solvent for the ${ }^{2} \mathrm{D}$, which can lead to a problem of mobile phase mismatch and poor or no separation in the ${ }^{2} \mathrm{D}$.

In particular, the analysis of polar compounds by an RPxHILIC coupling has been substantially less employed than the HILICxRP combination in the literature so far. The difficulty arises from the high HILIC sensitivity to the injection solvent, fractions coming from the ${ }^{1} \mathrm{D}$-RP solved in a high aqueous concentration are hardly focused in the ${ }^{2} \mathrm{D}$-HILIC causing a breakthrough phenomenon due to the nonretention of the transfer compounds in the ${ }^{2} \mathrm{D}$ column. This effect is even more negative when the injection volume exceeds the $9 \%$ of the column dead volume [3]. However, HILIC is able to provide fast analysis of compounds with a low loss of resolution [4] that can be a very interesting characteristic for the separation carried out in the ${ }^{2} \mathrm{D}$. So, the fast analysis provided by HILIC could be extrapolated to the analysis of polar compounds, which 2DLC analysis was mainly limited to the coupling of HILIC in the ${ }^{1} \mathrm{D}$ and RP in the ${ }^{2} \mathrm{D}$ up to now.

A big effort should be done in this kind of orthogonal coupling to avoid the mismatch solvent effect as far as possible. As a core part of a 2D-LC system, the modulator is responsible for the ${ }^{1} \mathrm{D}$ effluent cutting, collecting, and transferring to the ${ }^{2} \mathrm{D}$ column. The incompatibility of two dimensions will negatively affect the modulation process, resulting in analytes unfocussed on the head of the ${ }^{2} \mathrm{D}$ column. The tradition standard modulation (TS) is the most common and simple way to transfer the fractions from the ${ }^{1} \mathrm{D}$ to the ${ }^{2} \mathrm{D}$, and consists on the use of two identical injection loops installed in a two-position switching valve. These two loops continuously alternate their functions collecting the fractions from the ${ }^{1} \mathrm{D}$ and injecting them in the ${ }^{2} \mathrm{D}$ column. This modulation mechanism can be considered as a passive modulation since the fraction injected in the ${ }^{2} \mathrm{D}$ column is going to depend only on the ${ }^{1} \mathrm{D}$ flow rate and mobile phases. Due to this passive modulation, usually, the TS modulation produces a low focusing effect in the head of the ${ }^{2} \mathrm{D}$ column, because it is not able to reduce the strength of the fraction solvent before reaching the ${ }^{2} \mathrm{D}$ column.

Recently, several kinds of modulators have been developed to overcome the incompatibility problem [5]. Some of them are based on the modification of the modulator configuration like the Fixed Solvent Modulator (FSM) developed by Petersson et al. [6] or the Active Solvent Modulation (ASM) proposed by Stoll et al. [7, 8].
Besides the solvent incompatibility, LCxLC presents other limitations. For instance, due to the realistic limitation of the transferred volume from the ${ }^{1} \mathrm{D}$ to the ${ }^{2} \mathrm{D},{ }^{1} \mathrm{D}$ column diameter and flow rate are usually low in LCxLC and a small fraction is collected in the modulator which will be diluted by the ${ }^{2} \mathrm{D}$ high flow rate. Therefore, these limitations result in a hard detection of low abundant targets, i.e., low sensitivity. Based on the FSM modulation strategy, Chen's group made a new development to improve the ${ }^{2} \mathrm{D}$ detection sensitivity by replacing the sample loops with short $\mathrm{C} 18$ trapping columns to construct an RPLC $\times$ HILIC system [9], which is shown in Fig. S1 in the Electronic Supplementary Material (ESM). However, in all three described modulators, the fraction dilution factor depends on the length and inner diameter of the sample loop channel and the bypass channel or the length of the trapping and the splitting column. This fact involves precise control and optimization of the dilution factor during the analysis of a complex sample containing analytes with a wide range of polarity which can limit the method development.

As a further improvement, a novel modulator, called atcolumn dilution (ACD) modulator, was proposed in our previous work to achieve free adjustment of the online dilution factor [10]. The RPLCxHILIC configuration equipped with an ACD modulator is shown in Fig. 1. The ACD modulator was modified from the TS modulation (Fig. 1) by adding an independent transfer pump, which is responsible for the injection of the fractions into the ${ }^{2} \mathrm{D}$. In this modulation strategy, the ${ }^{2} \mathrm{D}$ mobile phase is no longer flowing through the sample loops, but the additional transfer pump is connected to the valve, and therefore, it is the responsible for eluting the ${ }^{1} \mathrm{D}$ fraction storage in the loop with a weaker solvent for the ${ }^{2} \mathrm{D}$ (transfer flow rate). Afterward, the transfer flow and the ${ }^{2} \mathrm{D}$ gradient flow meet and are mixed in a mixer before reaching the ${ }^{2} \mathrm{D}$ column. Thereby, the high water content fraction from the ${ }^{1} \mathrm{D}$-RP is diluted with acetonitrile and gradually enters the ${ }^{2} \mathrm{D}$ column, and the analytes can be focussed on the head of the ${ }^{2} \mathrm{D}$ column. More importantly, the dilution factor could be controlled by adjusting the transfer flow and the ${ }^{2} \mathrm{D}$ flow rates, since the transfer flow can be optimized regardless of the ${ }^{1} \mathrm{D}$ and ${ }^{2} \mathrm{D}$ conditions.

Herbal medicines are naturally occurring plant-derived substances, which are used for medical purposes [11]. According to the World Health Organization (WHO), about $80 \%$ of the world's population uses herbal medicines as its primary healthcare [12]. There is no doubt that herbal medicines have great medical potential and economical importance. However, the difficulty of obtaining a clear description of the complex chemical constituents limits the in-depth study of the efficacy principles and the quality control of these herbal medicines in practice. Therefore, the development of analytical methods and tools that allow the comprehensive analysis of the herbal medicines is highly demanded. However, due to the high complexity of the chemical constituents in 
Fig. 1 Configuration of the traditional standard (TS) modulator and at-column dilution (ACD) modulator. In this scheme, a 2position 8-port switching valve is shown to simplify the visualization of the new configuration

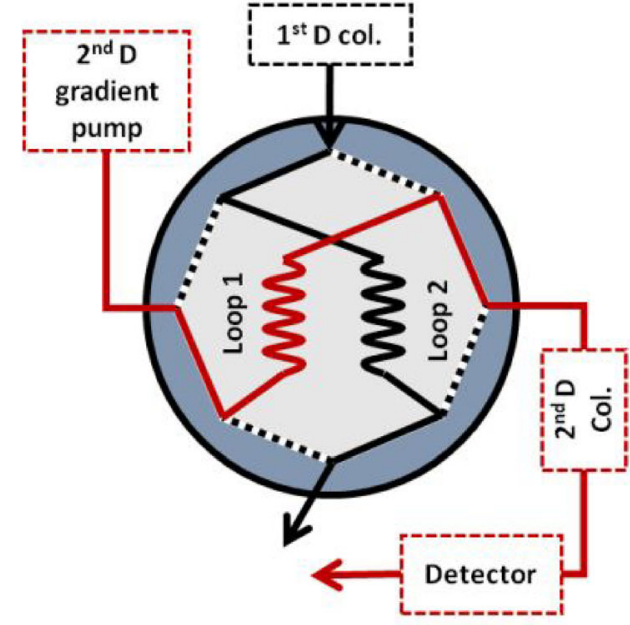

Traditional standard (TS) modulator

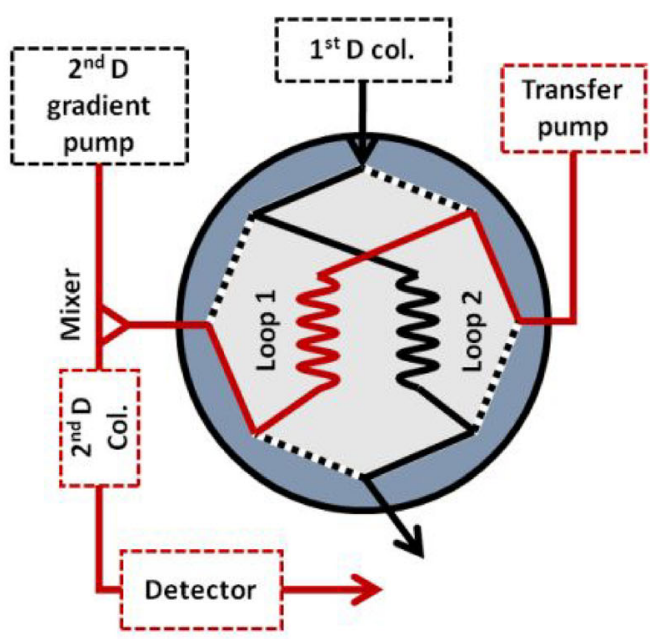

At Column Dilution ( $A C D$ ) modulator herbal medicines, conventional one-dimensional chromatographic techniques usually do not provide enough separation power to comprehensively analyze the chemical profile of such complex plants. Therefore, there is a motivation to seek more advanced analytical tools with higher separation performance.

Buddleja davidii is a multi-stemmed shrub or small tree that is native to China and has been introduced nowadays as an ornamental plant worldwide, both in sub-oceanic climates and sub-Mediterranean zones. In Germany, Buddleja davidii, commonly known as the butterfly bush, has very wide distribution due to its small wind-dispersed seeds rapidly colonize and barely disturb the area. It also grows widely due to its potential invasive power and adaptability.

Similar to other species of Buddleja, Buddleja davidii has significant pharmaceutical activities related to its metabolites. For example, terpenes (ESM Fig. S2a), phenylethanoids as verbascoside, shown in ESM Fig. S2b, which is known for its antioxidant, anti-inflammatory and photoprotective actions [13], and phenylpropanoid glycosides (ESM Fig. S2c), with antioxidant activities, also isolated and identified in this plant [14-16]. A flavone glycoside called linarin, shown in ESM Fig. S2d, was also isolated, and this compound revealed analgesic, antipyretic, anti-inflammatory, and neuroprotective activities [17]. Furthermore, a systematic survey of the genus Buddleja has indicated that some isolated compounds have anti-inflammatory and inhibitory activity against eicosanoid synthesis by inhibiting cyclo-oxygenase (COX) or 5lipoxygenase (5-LOX) [15, 18]. Therefore, a comprehensive study about its chemical constituents is highly required due to its rich and diverse potential medicinal value.

In this study, the applicability of ACD modulator is tested for the coupling of RPLCxHILIC-MS system. For this propose different parts of the herbal medicine Buddleja davidii were used as an example for the method development. As a starting point, careful optimization of ${ }^{1} \mathrm{D}$ and ${ }^{2} \mathrm{D}$ gradient separation was carried out for the root extract. Then, with the optimized 2D-LC separation conditions, four parts of Buddleja davidii were also analyzed. Finally, the 2D-LC-UV method was coupled to MS, and MS/MS experiments were carried out to identify the complex profile of the root sample.

\section{Experimental}

\section{Chemicals}

Acetonitrile (ACN, MS grade) was purchased from VWR International (USA). Formic acid (HPLC/SPECTRO grade) was from Merck KGaA (Germany) and ethanol (Analytical reagent grade) was purchased from Fisher Chemical (Fair Lawn, New Jersey, USA). Distilled water was filtered through a Sartorius Stedim Biotech system (Germany).

\section{Sample collection and preparation}

The whole Buddleja davidii plant was collected from the field around the University of Duisburg-Essen, Campus Essen, Germany. The whole plant was divided into five parts: roots, stems, leaves, flowers, and fruits. The roots were washed with distilled water and dried with a dryer at $45^{\circ} \mathrm{C}$ during $12 \mathrm{~h}$. The other four parts were directly dried in a dryer at $45^{\circ} \mathrm{C}$. Then, they were smashed into powder and preserved in brown glass bottles at room temperature. $5.0 \mathrm{~g}$ of each sample were extracted with $50 \mathrm{~mL} 60 \%$ ethanol for $1 \mathrm{~h}$ at room temperature in glass flasks, and then the samples were extracted in an ultrasonic bath (VWR, USA) for another $1 \mathrm{~h}$. After that, the samples were centrifuged (5840R Eppendorf, Germany) at 
$4000 \mathrm{rpm} / \mathrm{min}$ for $15 \mathrm{~min}$ at room temperature. The supernatant of the extract was moved to an ultracentrifuge (Spin plus from Eppendorf, Germany) and centrifuged at $14000 \mathrm{rpm} / \mathrm{min}$ for $10 \mathrm{~min}$ at room temperature. Afterward, $1 \mathrm{~mL}$ volume of the supernatants was diluted with $4 \mathrm{~mL}$ distilled water and the sample solution was filtered through a $0.2-\mu \mathrm{m}$ filter prior to 2D-LC analysis.

\section{Two-dimensional liquid chromatography (2D-LC) system}

An Agilent 1260 Infinity $\left({ }^{1} \mathrm{D}\right)$ combined with Agilent 1290 Infinity $\left({ }^{2} \mathrm{D}\right)$ was utilized as a $2 \mathrm{D}$ liquid chromatography system (Agilent Technologies, USA) in this study. In particular, the ${ }^{1} \mathrm{D}$ was built by a 1260 Infinity Flexible Cube solvent management module, a 1260 Infinity Degasser, a 1260 Infinity Capillary Pump, a 1260 Infinity Micro Autosampler, a 1260 Infinity Thermostat Column Compartment, a 1260 Infinity Diode Array Detector. The ${ }^{2} \mathrm{D}$ consisted of a 1290 Infinity II Binary Pump and a 1290 Infinity II Diode Array Detector. The interface or modulator consisted of a 1290 Infinity Valve Drive and a 2-position/4-port duo-valve equipped with two $80 \mu \mathrm{L}$ loops. The third pump, used for the ACD modulation was a 1290 Infinity binary pump. The 2DLC system was controlled by the OpenLab CDS software. The data of the diode array detector were collected during the running of the system and then imported to LC Image $2.7 \mathrm{r}$ $\mathrm{LC} \times \mathrm{LC}$ (64-bit) to generate 2D contour plots. The detection wavelength was set at $280 \mathrm{~nm}$ during the whole separation process.

The configuration of the RPLCxHILIC-MS system with the ACD modulator is shown on the right side of Fig. 1. At first, the mobile phase from the ${ }^{1} \mathrm{D}$ pump transferred the injected sample $(2 \mu \mathrm{L})$ to the RP- ${ }^{1} \mathrm{D}$ column. The ${ }^{1} \mathrm{D}$ effluent was alternatively stored in the sampling-loops of the electronically controlled 2-positon/4-port duo-valve, which hyphenated the ${ }^{1} \mathrm{D}$ column and the ${ }^{2} \mathrm{D}$ column. The switching time of the valve was set at $2 \mathrm{~min}$ (modulation time). Once the sample loop was filled with ${ }^{1} \mathrm{D}$ effluent, the valve changed the position and the fraction was connected in the line of ${ }^{2} \mathrm{D}$ flow, and then the collected fraction was pushed out of the sample loop by the transfer flow from the additional pump (transfer pump). At downstream of the modulator, the transfer flow-with a relative low flow rate - and the ${ }^{2} \mathrm{D}$ eluent-with a high flow rate - met and mixed in a mixer and were transferred into the ${ }^{2} \mathrm{D}$ column. Finally, after the detection in the ${ }^{2} \mathrm{D}$ UV-detector, the ${ }^{2} \mathrm{D}$ effluent was split to enter into the MS. In this work, the optimized transfer flow rate was $0.2 \mathrm{~mL} / \mathrm{min}$ and the ${ }^{2} \mathrm{D}$ flow rate was established at $2.4 \mathrm{of} \mathrm{mL} / \mathrm{min}$, which lead to a total ${ }^{2} \mathrm{D}$ flow rate of $2.6 \mathrm{~mL} / \mathrm{min}$ and a dilution factor of 13 (total flow rate/transfer flow rate).

${ }^{1} \mathrm{D}$ RP separation was performed at room temperature on a C18 column $(150 \times 1.0 \mathrm{~mm})$ with $2.6 \mu \mathrm{m}$ core-shell particles
(Phenomenex, CA, USA). The mobile phase contained water (A) and acetonitrile with $0.1 \%$ formic acid (B). The optimized gradient time was $70 \mathrm{~min}$ at a flow rate of $15 \mu \mathrm{L} / \mathrm{min}$. The gradient consisted of: $0 \mathrm{~min}, 10 \% \mathrm{~B} ; 3 \mathrm{~min}, 20 \% \mathrm{~B} ; 20 \mathrm{~min}$, $20 \% \mathrm{~B}$; $36 \mathrm{~min}, 45 \%, 50 \mathrm{~min}, 100 \% \mathrm{~B} ; 64 \mathrm{~min}, 100 \% \mathrm{~B}$; 65 min, $10 \%$ B (Fig. 2).

${ }^{2} \mathrm{D}$ HILIC separation was performed at room temperature on a Cys-HILIC column $(150 \times 3.0 \mathrm{~mm})$ packed with $5 \mu \mathrm{m}$ particles (Acchrom, Inc., Beijing, China). The mobile phase contained water with $0.1 \%$ formic acid (A) and acetonitrile (B). The modulation time was 2 min, which leads to 35 cycles presented in the 2D separation. As shown in Fig. 2, the lowest point of the ${ }^{2} \mathrm{D}$ shifting gradient in each cycle increases in 70 min from 65 to $75 \%$ of acetonitrile (B). The gradient was established as follow: $0 \mathrm{~min}, 100 \% \mathrm{~B} ; 0.1 \mathrm{~min}, 0.1 \% \mathrm{~B}$; $0.2 \mathrm{~min}, 95 \% \mathrm{~B} ; 1.5 \mathrm{~min}, 75 \% \mathrm{~B}$ (at the beginning) or $65 \%$ $\mathrm{B}$ (at the end); $1.6 \mathrm{~min}, 75 \% \mathrm{~B}$ (at the beginning) or $65 \% \mathrm{~B}$ (at the end), $1.7 \mathrm{~min}, 100 \% \mathrm{~B} ; 100 \%$ was maintained until $2 \mathrm{~min}$ for the equilibration of the column before the start of the next measurement.

\section{Mass spectrometry}

Mass spectrometry was performed on an Agilent 6545 Q-TOF LC/MS (Agilent Technologies, USA) using an electrospray ionization source (ESI) operating in negative ion mode. A flow of $0.4 \mathrm{~mL} / \mathrm{min}$ was introduced into the ion source of the MS by splitting the ${ }^{2} \mathrm{D}$ flow rate with a T-valve. Nitrogen was utilized as sheath gas and drying gas. The sheath gas and the drying gas were set to $11 \mathrm{~L} / \mathrm{min}$ at $300{ }^{\circ} \mathrm{C}$ and $10 \mathrm{~L} / \mathrm{min}$ at $350^{\circ} \mathrm{C}$, respectively. The nebulizer was set at 35 psig and the capillary voltage and nozzle voltage were set at $3500 \mathrm{~V}$ and $1000 \mathrm{~V}$, respectively. The fragmentor, skimmer, and Oct 1 RF Vpp were set at $220 \mathrm{~V}, 65 \mathrm{~V}$, and $750 \mathrm{~V}$, respectively. The acquisition mode was performed on full MS and auto MS/MS. The acquisition was performed in full scan and automatic MS/MS, at $1 \mathrm{spectra} / \mathrm{s}$, in the $\mathrm{m} / \mathrm{z}$ range $100-1500$ (MS) or 100-1300 (MS/MS). The collision energy was $40 \mathrm{eV}$. The MS system was controlled by the software Agilent Mass Hunter Workstation Data Acquisition. Then the data could be analyzed by Agilent Mass Hunter Qualitative Analysis Navigator and imported into LC Image 2.7r LC $\times$ LC (64bit) to generate MS contour plots.

\section{Results and discussion}

\section{Optimization of the gradient for the ${ }^{1} D$ (RPLC) separation}

The different parts of Buddleja dividii were expected to present similar metabolite families, therefore, the root was chosen to optimize the ${ }^{1} \mathrm{D}$ RP separation gradient. Several gradients 
Fig. 2 Optimized 2D-LC gradient for ${ }^{1} \mathrm{D}$ and ${ }^{2} \mathrm{D}$ separation

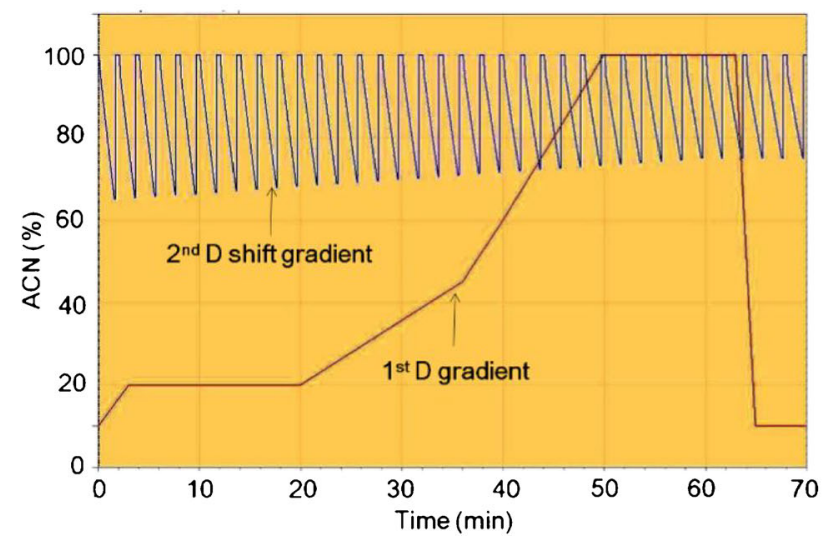

were tested, the detailed information about these gradients is listed in ESM Table S1. Figure 3 shows the chromatograms of the root extract separation obtained with the listed gradients. At first, to measure the polarity distribution of all constituents, a full gradient with ACN as organic solvent from 5 to $90 \%$ in 55 min was performed as shown in Fig. 3a. Most of the peaks were overlapped without being well separated in the time interval between 20 and 25 min. To perform a better separation of all compounds, a de novo optimization strategy was applied. In this strategy, the optimization was consequently performed segment by segment from the beginning. When the separation of analytes in a segment was optimized, the analytes in the next segment were eluted directly with a high content of organic phase to save time. By analogy, the optimized segment was fixed and the next segment continued to be optimized until the separation optimization of all analytes was completed (Fig. 3a-e). As shown in Fig. 3e, after only four steps, an evenly distributed separation was achieved with
Fig. 3 Optimization of the gradient for the ${ }^{1} \mathrm{D}$ RP separation

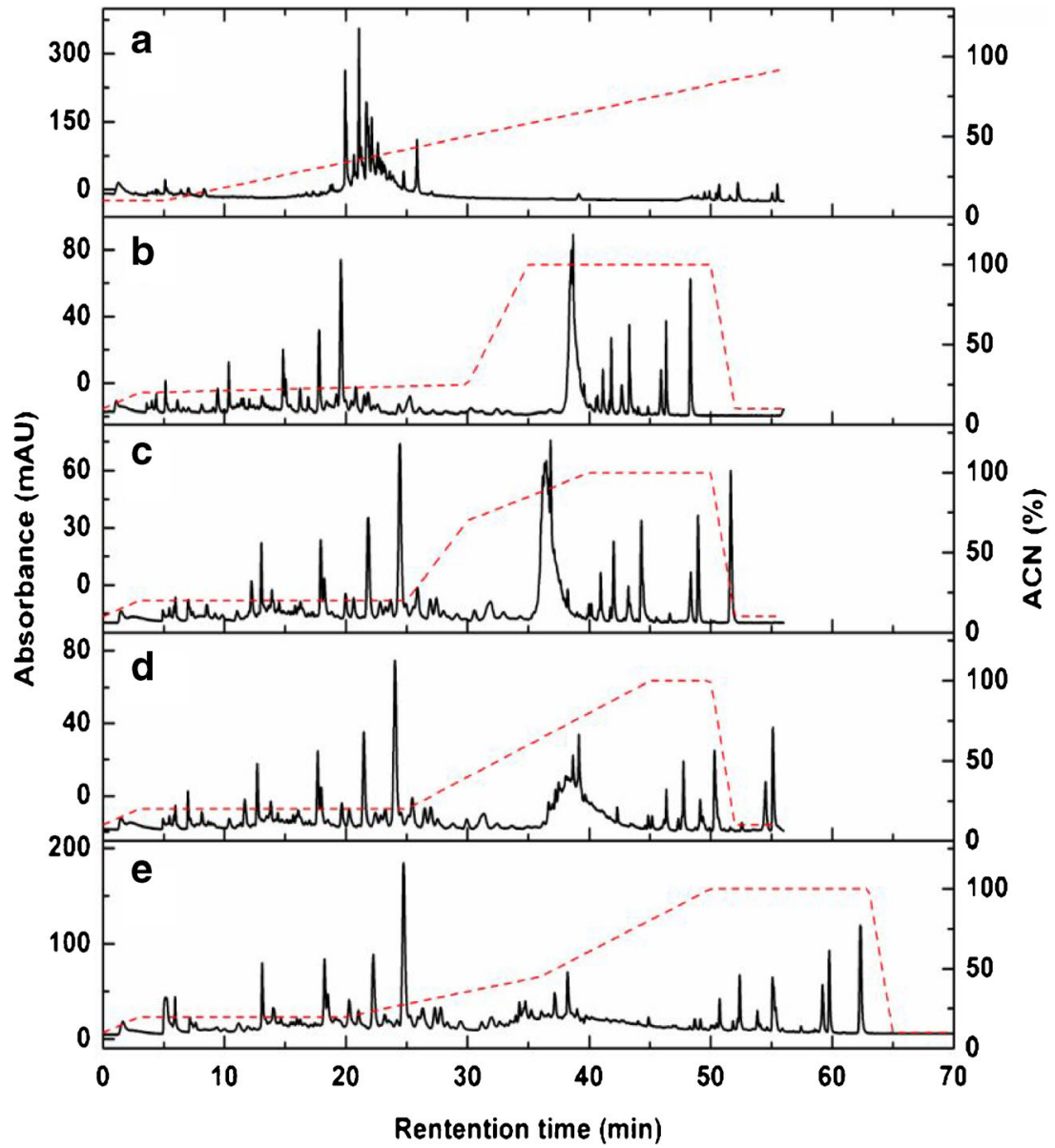


a final analysis time of $70 \mathrm{~min}$. Therefore, the gradient showed in Fig. 3e was applied in the following experiments as an optimized ${ }^{1} \mathrm{D}$ gradient.

\section{Optimization of the gradient for the ${ }^{2} D$ (HILIC) separation}

The composition of mobile phase in the early stage of ${ }^{2} \mathrm{D}$ gradient cycle is critical for focusing the transfer analytes on the head of the ${ }^{2} \mathrm{D}$ column. Any modification of the ${ }^{2} \mathrm{D}$ gradient in this stage might change the separation in ${ }^{2} \mathrm{D}$. Therefore, the optimization of the ${ }^{2} \mathrm{D}$ HILIC gradient will focus on the early stage of the gradient cycle.

In this term, as a first and common strategy, we installed $80 \mu \mathrm{L}$ loops in the interface. Considering the ${ }^{1} \mathrm{D}$ flow rate (15 $\mu \mathrm{L} / \mathrm{min})$ and the modulation time $(2 \mathrm{~min})$, loops of $30 \mu \mathrm{L}$ volume would be enough for the collection of the whole ${ }^{1} \mathrm{D}$ fraction; however, with the use of larger loops $(80 \mu \mathrm{L})$ connected to the interface, $50 \mu \mathrm{L}$ of the loops was filled with ${ }^{2} \mathrm{D}$ mobile phase, which allowed diluting the ${ }^{1} \mathrm{D}$ fraction in a more adequate solvent, as demonstrated by Montero et al. [19]. This fact contributed to reduce the mismatch solvent incompatibility together with the ACD modulation, helping the focusing effect in the head of the ${ }^{2} \mathrm{D}$ column.

In this regard, the effect of the injection volume in relation to the column void volume of the ${ }^{2} \mathrm{D}$ column is a critical point in online LCxLC, mainly due to the combination of the ${ }^{1} \mathrm{D}$ solvent strength and the very fast flow rates typically used in the ${ }^{2} \mathrm{D}$ may hamper the performance of the separation of the analytes solved in the injected fraction, producing a bandbroadening effect. Typically, the injection volume in the ${ }^{2} \mathrm{D}$ column (loop size) should not exceed the $15 \%$ of the column void volume to reduce the band-broadening negative effects [20]; however, in the case of HILIC, the recommendation is much stricter considering the important effect of the injection solvent on the HILIC separation. Some results recommend that the injection volume should not exceed the $9 \%$ of the column void volume [3] but others reduce this percentage until the $1 \%$ [21]. In this work, when the TS modulator was employed, the injection volume $(80 \mu \mathrm{L})$ implied the $11 \%$ of the ${ }^{2} \mathrm{D}$ column volume, considering the dimensions of the ${ }^{2} \mathrm{D}$ column $(150 \times 3 \mathrm{~mm}, 5 \mu \mathrm{m})$ and the interstitial column volume ( $68 \%$ of the total volume), which determine a dead volume of the ${ }^{2} \mathrm{D}$ column of $720.8 \mu \mathrm{L}$. However, with ACD modulation, there is not a real injection volume as it happens in the TS modulation, since in ACD the transfer pump elutes the fraction from the loop with a controlled flow rate (slower than the ${ }^{2} \mathrm{D}$ pump flow rate used in the TS modulator configuration for the elution of the fraction collected in the loop) and after that, this flow rate is mixed with the high ${ }^{2} \mathrm{D}$ flow rate, so in this case, we can talk about a transfer fraction diluted by two different flow rates more than an injection volume, which improves even more the focusing effect in the head of the ${ }^{2} \mathrm{D}$ column.

Moreover, the use of shifting gradients along the LCxLC analysis not only helps to achieve more orthogonality but also to reduce the breakthrough since a modification of the ${ }^{2} \mathrm{D}$ initial mobile phase conditions can be set accordingly to the ${ }^{1} \mathrm{D}$ eluent. Fig. $4 \mathrm{~b}, \mathrm{~d}$, f, and h show four different ${ }^{2} \mathrm{D}$ shift gradients, in which $\alpha$ and $\beta$ represent the first gradient cycle and the last gradient cycle, respectively in the ${ }^{2} \mathrm{D}$ shift gradients. In Fig. 4a, c, e, and g, the corresponding separations are shown. At the beginning and the end of the $4 \mathrm{~b}$ and $4 \mathrm{~d}$ gradients, the organic content of ACN was set at $95 \%$ and $100 \%$, respectively. The separations obtained using these two gradients are shown in Fig. 4a and c. Although theoretically, under HILIC mode separation a small aqueous percentage is needed to create a water-layer around the polar particles, comparing both conditions, using $100 \% \mathrm{ACN}$ as initial conditions the retention of the compounds was significantly increased (Fig. 4d). In addition, the peak width in zone c (Fig. 4c) was much narrower than that in zone a (Fig. 4a). This indicates that a $100 \%$ ACN used at the beginning and at the end of the fast ${ }^{2} \mathrm{D}$ gradient leads to a better separation. Applying higher percentage of $\mathrm{ACN}$ at the end of the gradient would accelerate the equilibrium of the ${ }^{2} \mathrm{D}$ HILIC column and prepare the conditions for the injection of the next cycle. Furthermore, applying higher content of ACN at the beginning of the gradient would improve the dilution effect of transferred fractions under ACD modulation mode, which results in better focus of analytes on the head of the ${ }^{2} \mathrm{D}$ HILIC column. However, as can be observed in Fig. 4c, the separation with the gradient $4 \mathrm{~d}$ was not optimal since the main part of the peaks were separated within a narrow small ${ }^{2} \mathrm{D}$ area. The adjustment of the gradient was performed based on the gradient shown in Fig. 4d as shown in Fig. 4f and h. A rapid decrease of ACN to 95\% (Fig. 4f) or 90\% (Fig. 4h) was set in each cycle between 0.2 and $0.25 \mathrm{~min}$. The chromatogram shown in Fig. $4 \mathrm{f}$ indicates that $5 \%$ decrease of ACN resulted in a slightly higher coverage of the $2 \mathrm{D}$ space in comparison with the distribution achieved using gradient $4 \mathrm{~h}$.

It is worth to point out that serious breakthrough was observed in the $2 \mathrm{D}$ contour plot. This might be attributed to a large amount of low polar compounds in the roots, which were hardly retained on the ${ }^{2}$ D HILIC column. Nevertheless, any combination would have limitations. In this case, the combination of RPLC/HILIC is appropriated for the separation of amphiphilic compounds but is not suitable enough for the separation of homophilic compounds. The target of this work would focus on the amphiphilic compounds rather than homophilic compounds. Therefore, gradient $4 \mathrm{f}$ was determined as the optimal ${ }^{2} \mathrm{D}$ gradient used for the LCxLC system for the following measurements. 

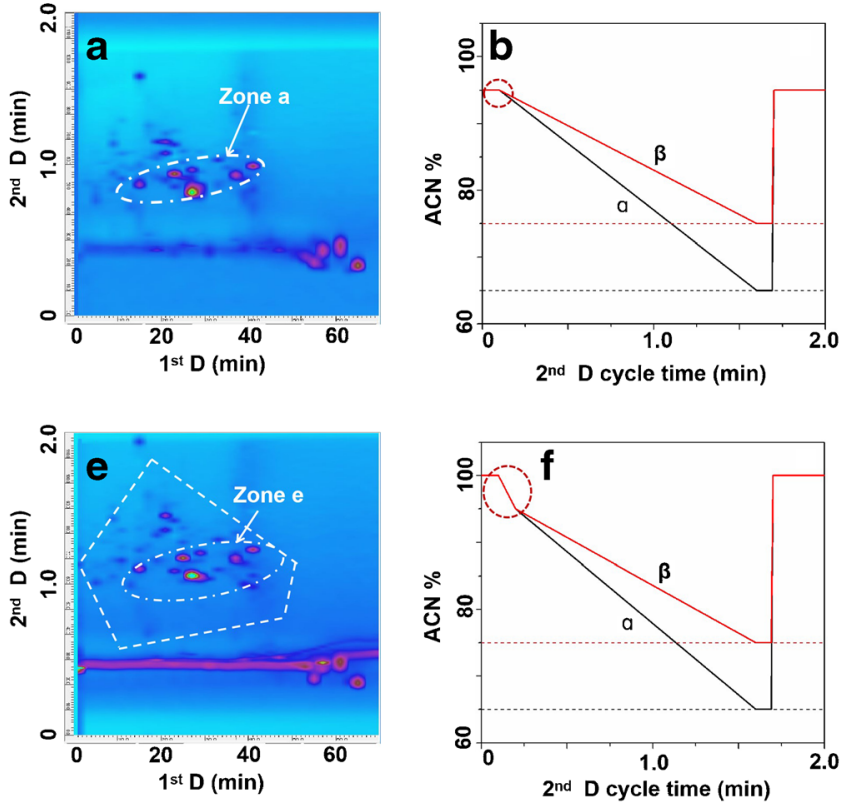

Fig. 4 Optimization of the ${ }^{2} \mathrm{D}$ shift gradient ( $\alpha$, first gradient cycle and $\beta$, last gradient cycle in ${ }^{2} \mathrm{D}$ shift gradient). a $2 \mathrm{D}$ plot of the root separation using the gradient shown in $\mathbf{b}(95 \%$ of mobile phase B as initial conditions in the ${ }^{2} \mathrm{D}$ gradients). $\mathrm{c} 2 \mathrm{D}$ plot of the root separation using the gradient shown in $\mathbf{d}$ ( $100 \%$ of mobile phase B as initial conditions in the ${ }^{2} \mathrm{D}$ gradients). e $2 \mathrm{D}$ plot of the root separation using the gradient

\section{RPLCXHILIC for different parts of Buddleja davidii}

With the optimized ${ }^{1}$ D RP gradient and ${ }^{2}$ D HILIC shift gradient, the separation of the other four parts of Buddleja davidii including flower, leaf, stem, and fruit were performed on the RPLCxHILIC system with ACD modulation. The results are shown in Fig. 5a, b, e, and f. Due to the high-efficiency dilution of the transferred fraction, the constituents of the Buddleja davidii extracts were well separated into narrow and symmetric peaks over the ${ }^{2} \mathrm{D}$ analysis. Compared to Fig. $5 \mathrm{a}, \mathrm{b}$, and $\mathrm{f}$, a serious breakthrough could be observed in Fig. $5 \mathrm{e}$. This result indicates that there are many homophilic compounds existing in the stem, which is similar to the result obtained in root separation. The results shown in Fig. 5a and $\mathrm{b}$ also indicate that more polar compounds with high concentration could be observed in the flowers and leaves than in other parts, which also implies that the metabolic profiles and, therefore, the metabolic activities of flowers and leaves are more vigorous than in other parts.

To demonstrate the advantage of the ACD modulation, the separation of the flower and leaf extract was also performed with TS modulation under the optimized gradient, the separation results are shown in Fig. 5c and d. Compared to the separation shown in Fig. 5a and b, without ACD modulation much less peaks could be separated and they presented lower peak intensities. This negative effect occurred because of the no-dilution of the fraction in the TS modulation which
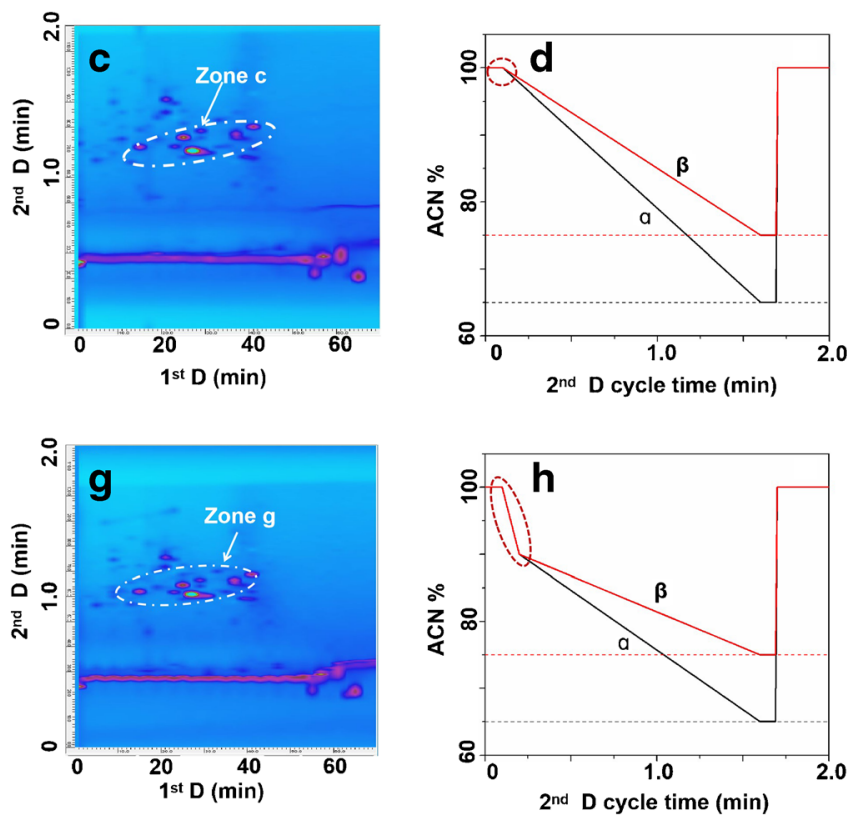

shown in $\mathbf{f}(100 \%$ of mobile phase B followed by a second step reducing the mobile phase $\mathrm{B}$ as initial conditions in the ${ }^{2} \mathrm{D}$ gradients). $\mathrm{g}$ 2D plot of the root separation using the gradient shown in $\mathbf{h}(100 \%$ of mobile phase $\mathrm{B}$ followed by a second step with a faster reduction of mobile phase $\mathrm{B}$ as initial conditions in the ${ }^{2} \mathrm{D}$ gradients)

resulted in a considerable breakthrough in the dead volume. The solvent in the transferred fraction (high content of water) was a very strong elution solvent for the ${ }^{2}$ D HILIC separation, which prevents a focussing effect of the analytes on the head of the ${ }^{2}$ D HILIC column. In conclusion, the ACD modulation provides much better flexibility than the TS modulation for the dilution of the transferred fraction and the achievement of the re-focusing and ${ }^{2} \mathrm{D}$ separation.

\section{Constituents analysis for the root of Buddleja davidii by RPLCXHILIC-Q-TOF MS}

\section{The advantage of RPLCXHILIC coupled to MS with ACD modulation}

For further evaluation of the established RPLCxHILIC method, two 2D-LC configurations with and without ACD modulation were coupled to DAD and Q-TOF MS detectors to study the chemical constituents in the Buddleja davidii root. The high effective separation power provided by the ACD modulation facilitates the online identification of the constituents in root by coupling 2D-LC to high-resolution mass spectrometry. The results are shown in ESM Fig. S3. Figs. S3A and S3E show the UV contour plots for both measurements. As previously discussed, serious breakthrough could be observed at the dead volume without ACD modulation (ESM Fig. S3A), and much more improvement was achieved with 
Fig. 5 Contour plots of

RPLCxHILIC with ACD

modulation or with TS

modulation for different parts of

Buddleja davidii. Flower with

ACD modulation (a), leaf with

ACD modulation (b), leaf with

TS modulation (c), flower with

TS modulation (d), stem with

ACD modulation (e), fruit with

ACD modulation (f)
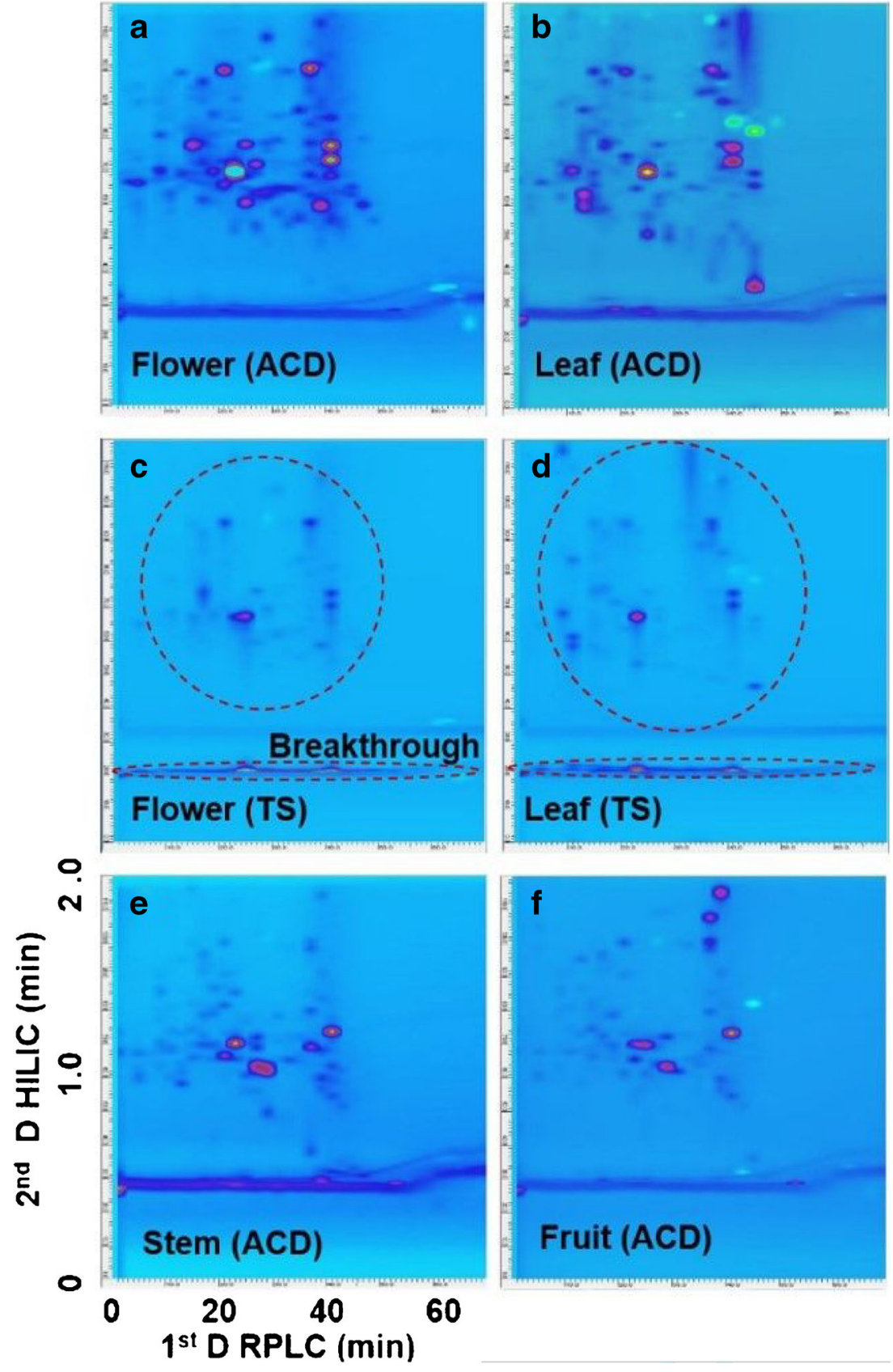

60
ACD modulation (ESM Fig. S3E). The measurement without ACD modulation showed that only a few peaks could be well retained and observed in the $2 \mathrm{D}$ contour plot, which means that most constituents were eluted with the transferred fraction solvent in the ${ }^{2} \mathrm{D}$ system without retention on the ${ }^{2} \mathrm{D}$ HILIC stationary phase.

The same result could be obtained from the extracted ion chromatogram (EIC) contour plots in ESM Fig. S3. As observed from the EIC contour plots, with ACD modulation (ESM Figs. S3F, G and H), the spots show very good shapes and a clear background without any breakthrough, which can be attributed to the sufficient dilution of the transferred fraction. This leads to highly focus of amphiphilic analytes on the head of the ${ }^{2}$ D HILIC column. In contrast, with TS modulation, a significant peak splitting and fronting were found in the EIC contour plots (Figures S3B, C and D). It indicated that the amphiphilic compounds were divided in the ${ }^{2} \mathrm{D}$ analysis into a non-retained portion and a retained portion. The compounds from the non-retained portion were quickly eluted together with the high water content solvent from the transferred fraction, forming a breakthrough peak in the dead volume due to a scarce dilution during the modulation. However, in the retained portion, part of the amphiphilic compounds presented relative separation in the ${ }^{2} \mathrm{D}$ analysis 
probably due to an inter-diffusion effect in the loop between the transferred fraction and the ${ }^{2} \mathrm{D}$ mobile phase, that allowed a partial focusing in the ${ }^{2} \mathrm{D}$ column.

Nevertheless, the insufficient dilution produced by the inter-diffusion in the loop still resulted in serious peak fronting. As a serious consequence, the existence of the splitting peaks at dead volume might lead to a misunderstanding on the number of isomers, as shown in the EIC contour plot in ESM Figs. S3B, C and D, which could be considered as double when TS modulation is applied. Those results indicate that enough dilution of the transferred faction in high orthogonality $2 \mathrm{D}$-LC is necessary to achieve proper separation and true MS chromatogram, avoiding misinformation.

The results of the EIC contour plots with ACD modulation demonstrate that the coupling of the proposed 2D-LC system with high-resolution mass spectrometry could provide highquality MS information for the online identification of chemical components from complex samples.

\section{Qualitative analysis of constituents in the roots of Buddleja davidii}

The MS and MS/MS measurements of the root extract were performed with the optimized 2D-LC-UV-MS method. The general method for the identification of compounds in the sample mixture was carried out by comparing the detected molecular ion and MS/MS fragment ions with the reported information in the literature. Therefore, the reported researches about Buddleja davidii were carefully reviewed. Firstly, the comparison of the theoretically calculated molecular ion masses of the reported compounds with the detected molecular ion masses obtained by Q-TOF was done. Once the detected mass value was the same as the calculated ion mass of the reported compounds, it was postulated that the same compound was detected in the prepared sample. However, without MS/MS information and only with the sum formula is hardly possible to carry out an identification due to a multitude of possible isobaric compounds. Therefore, MS/MS analysis was performed. Even with MS/MS data, uncharacteristic fragmentation is often difficult to interpret and, besides, the differentiation between isomeric compounds is not possible. Here, the combination of ion mobility with MS could be helpful [22].

Due to the lack of previous information about the chemical composition of this plant, only a few compounds with defined structures were found in the reported work. To reduce this problem, another strategy was developed to characterize as much Buddleja davidii compounds separated by the 2D-LC-MS analysis as possible. According to the principles of biology, the plants with a genetic relationship usually have similar metabolic networks, resulting in similar chemical constituents. Based on this, in addition to the reported information of the compounds in Buddleja davidii, the components with defined structure in various species of the genus Buddleja were collected to extend the candidate compounds. As shown in ESM Fig. S1, in the literature several compounds of the genus Buddleja were described and these were considered possible candidates for Buddleja davidii [13-18, 23-29]. The accurate molecular ion masses $[\mathrm{M}-\mathrm{H}]^{-}$of each compound collected from the information about the Buddleja genus were calculated with Agilent MassHunter Qualitative Analysis Navigator software. Then the data were imported to the software LC Image. With this software, the contour plots can be easily generated, which allows the determination of a given molecular ion masses $[\mathrm{M}-\mathrm{H}]^{-}$existing in the huge total ion current (TIC) mass information. According to the calculated molecular ion masses $[\mathrm{M}-\mathrm{H}]^{-}$of the Buddleja described compounds, the EIC contour plots were extracted from the TIC contour plot.

After that, the compounds that were not found in the analysis were excluded. For example, two typical compounds present in the genus Buddleja have an $m / z 283.0612$ and 423.3632, which correspond to acacetin and $\delta$-amyrone, respectively. The EIC of both compounds in the RPxHILIC analysis of the root is shown in ESM Fig. S4. In the EIC contour plot of $m / z 283.0612$, a clear spot was observed, which means that this compound is presented with a relative high concentration. In contrast, in the contour plot of the $m / z 423.3632$, there is no clear spot, but a complex background was observed, which means that the ion with $\mathrm{m} / \mathrm{z}$ 423.3632 was not present in Buddleja davidii root or its intensity was too low to be detected. Thus, this ion would be ignored in this study.

Obviously, in the TIC, many more ions existed than those we screened with the above method. In order to find out more ions with relatively high concentrations, further effort was done with the LC image software by comparing the UV and TIC contour plots. On one hand, the positions of the signals in the TIC contour plot were compared with the spots in the UV contour plot, and carefully examined to find out new ions, and then the EIC contour plot was generated and saved in LC image software. On the other hand, the spots observed in the TIC contour plot, but not in the UV contour plot, were also checked. After that, the ions in the EIC contour plot were further selected and excluded according to the method shown in ESM Fig. S4. Considering the molecular mass and the MS/MS fragments, these compounds were also tentatively identified.

Finally, EIC contour plots, which were screened by the previous three methods, were merged. As shown in ESM Fig. S5, some EIC contour plots contained one spot, for example in the $m / z 905.2727$ (ESM Fig. S5 left panel). Other EIC contour plots might contain several spots for the same mass, which indicated the existence of multiple isomers such as $m / z 637.2148$ (ESM Fig. S5 right panel). For both situations, the molecular ions and corresponding MS/MS fragments, once detected, were collected and then listed in Table 1. In total, 45 compounds including isomers have been found in the Buddleja davidii root sample. However, due to scarce available information about the chemical composition 


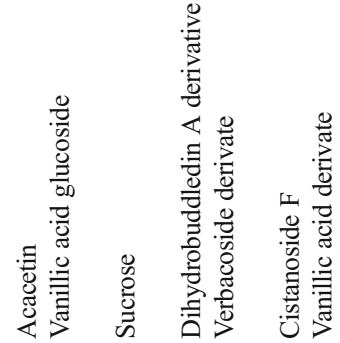

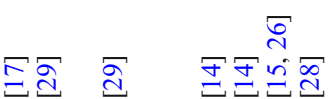

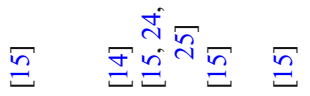

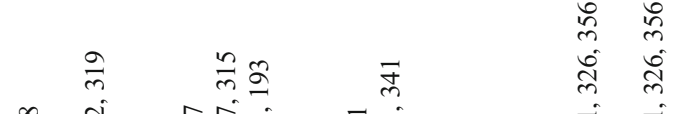

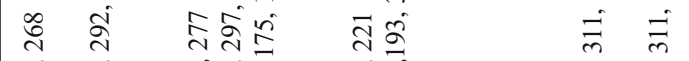

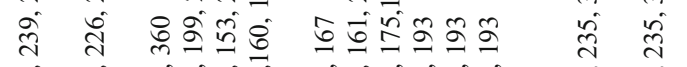

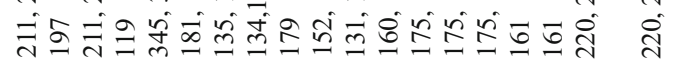

मी

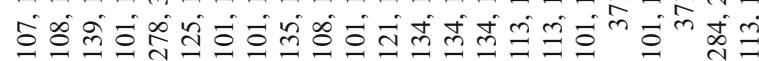

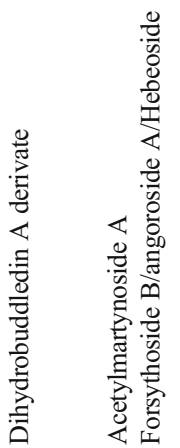

ह6.

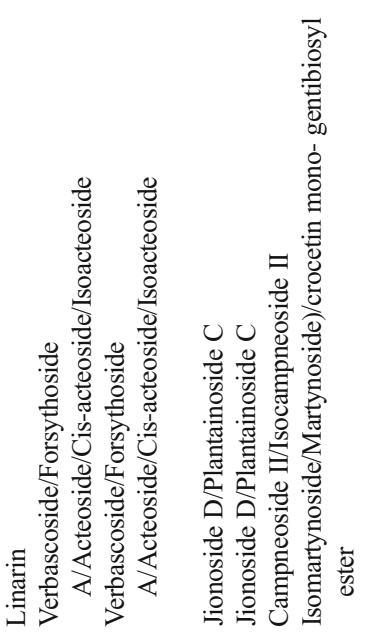

$\sigma \underline{6}$

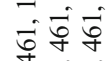

ู่อูล

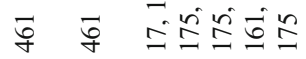

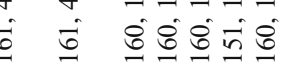

ली लिखिय

$\stackrel{1}{=}$

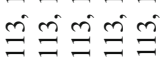

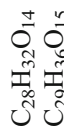

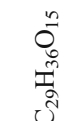

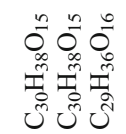

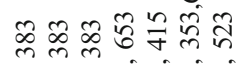

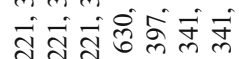

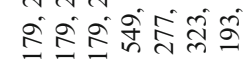

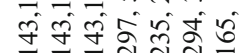

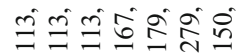

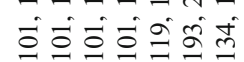

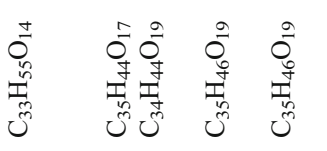

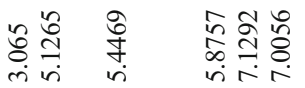

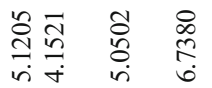

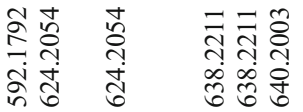

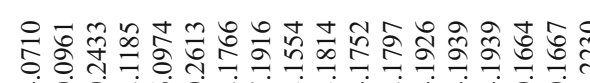

\section{तิ}

$\stackrel{\infty}{\infty}$

\section{$\infty$}

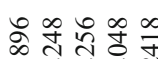

तై

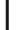

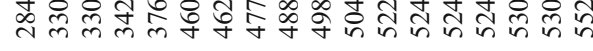

in कूष

पुँ

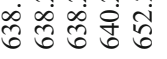

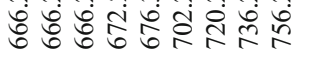

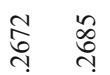

융 훙

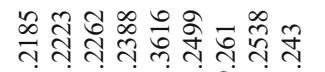

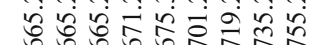

$\stackrel{2}{\lessgtr}$ 
of this plant, some ions could not be identified accurately by comparing to the detected ions with the reported works.

\section{Conclusion}

In this study, a recent developed RPLCxHILIC system with at-column dilution (ACD) modulation was applied for a comprehensive analysis of constituents in Buddleja davidii. With ACD modulation, the incompatibility of high orthogonal 2DLC systems could be successfully overcome. Meanwhile, the flexible adjustment of the dilution of the transferred fraction supported the advantages of the RPLCxHILIC coupling for an excellent separation of amphiphilic compounds with a wide range of polarity. The results demonstrated the potential of a 2D-LC system with ACD modulation for wide applications in the herbal medicine analysis. With this method, a broad separation of the metabolites present in Buddleja davidii was achieved. However, more information about the chemical composition of the separated compounds should be done to obtain comprehensive information about the metabolic profile of this herbal medicinal. Nevertheless, the detailed MS and MS/MS data of some detected ions with relative high intensities can be considered as a good reference for further studies.

Acknowledgements Open Access funding provided by Projekt DEAL.

Funding information This work was financially supported by the China Scholarship council (CSC).

\section{Compliance with ethical standards}

Conflict of interest The authors declare that they have no conflict of interest.

Open Access This article is licensed under a Creative Commons Attribution 4.0 International License, which permits use, sharing, adaptation, distribution and reproduction in any medium or format, as long as you give appropriate credit to the original author(s) and the source, provide a link to the Creative Commons licence, and indicate if changes were made. The images or other third party material in this article are included in the article's Creative Commons licence, unless indicated otherwise in a credit line to the material. If material is not included in the article's Creative Commons licence and your intended use is not permitted by statutory regulation or exceeds the permitted use, you will need to obtain permission directly from the copyright holder. To view a copy of this licence, visit http://creativecommons.org/licenses/by/4.0/.

\section{References}

1. Liu Y, Xue X, Guo Z, Xu Q, Zhang F, Liang X. Novel twodimensional reversed-phase liquid chromatography/hydrophilic interaction chromatography, an excellent orthogonal system for practical analysis. J Chromatogr A. 2008;1208:133-40. 
2. François I, Sandra K, Sandra P. Comprehensive liquid chromatography: fundamental aspects and practical considerations-a review. Anal Chim Acta. 2009;641:14-31.

3. D'Attoma A, Heinisch S. On-line comprehensive two dimensional separations of charged compounds using reversed-phase high performance liquid chromatography and hydrophilic interaction chromatography. Part II: application to the separation of peptides. $\mathrm{J}$ Chromatogr A. 2013;1306:27-36.

4. Chauve B, Guillarme D, Cléon P, Veuthey JL. Evaluation of various HILIC materials for the fast separation of polar compounds. J Sep Sci. 2010;33:752-64.

5. Chen Y, Montero L, Schmitz OJ. Advance in on-line two-dimensional liquid chromatography modulation technology. Trends Anal Chem. 2019;120:115647.

6. Petersson P, Haselmann K, Buckenmaier S. Multiple heart-cutting two dimensional liquid chromatography mass spectrometry: towards real time determination of related impurities of biopharmaceuticals in salt based separation methods. J Chromatogr A. 2016;1468:95-101.

7. Stoll DR, Shoykhet K, Petersson P, Buckenmaier S. Active solvent modulation: a valve-based approach to improve separation compatibility in two-dimensional liquid chromatography. Anal Chem. 2017;89:9260-7.

8. Stoll DR, Harmes DC, Staples GO, Potter OG, Dammann CT, Guillarme D, et al. Development of comprehensive online twodimensional liquid chromatography/mass spectrometry using hydrophilic interaction and reversed-phase separations for rapid and deep profiling of therapeutic antibodies. Anal Chem. 2018;90: 5923-9.

9. Chen YZ, Wu YJ, Liu XM, Li BW, Hu DP, Huang S, et al. Pulsed elution modulation for on-line comprehensive two-dimensional liquid chromatography coupling reversed phase liquid chromatography and hydrophilic interaction chromatography. J Chromatogr A. 2019;1583:98-107.

10. Chen Y, Li J, Schmitz OJ. Development of an at-column dilution modulator for flexible and precise control of dilution factors to overcome mobile phase incompatibility in comprehensive twodimensional liquid chromatography. Anal Chem. 2019;91:10251-7.

11. Tilburt JC, Kaptchuk TJ. Herbal medicine research and global health: an ethical analysis. Bull World Health Organ. 2008;86: 594-9.

12. Sardesai VM. Herbal medicines: poisons or potions? J Lab Clin Med. 2002;139:343-8.

13. Vertuani S, Beghelli E, Scalambra E, Malisardi G, Copetti S, Toso $\mathrm{RD}$, et al. Activity and stability studies of verbascoside, a novel antioxidant, in dermo-cosmetic and pharmaceutical topical formulations. Molecules. 2011;16.

14. Yamamoto A, Nitta S, Miyase T, Ueno A, Li-Jun W. Phenylethanoid and lignan-iridoid complex glycosides from roots of Buddleja davidii. Phytochemistry. 1993;32:421-5.

15. Liao Y, Houghton PJ, Hoult JRS. Novel and known constituents from buddleja species and their activity against leukocyte eicosanoid generation. J Nat Prod. 1999;62:1241-5.

16. Ahmad M, Sticher O. Isolation of acacetin-7-O-rutinoside and martynoside from Buddleja davidii. J Chem Soc Pak. 1988;10: 117-23.

17. Feng X, Wang X, Liu Y, Di X. Linarin inhibits the acetylcholinesterase activity in-vitro and ex-vivo. Iran J Pharm Res. 2015;14:949-54.
18. Hoult JRS, Moroney MA, Payá M. Actions of flavonoids and coumarins on lipoxygenase and cyclooxygenase. Method Enzymol. 1994;234:443-54.

19. Montero L, Herrero M, Prodanov M, Ibáñez E, Cifuentes A. Characterization of grape seed procyanidins by comprehensive two-dimensional hydrophilic interaction $\times$ reversed phase liquid chromatography coupled to diode array detection and tandem mass spectrometry. Anal Bioanal Chem. 405:4627-38.

20. Dolan JW. How much can I inject? Part II: injecting in solvents other than mobile phase. LC-GC. 2014:854-9.

21. Patrik Appelblad, Tobias Jonsson, Einar Pontén, Viklund C, Jiang. W. A practical guide to HILIC. Editor: Tobias Jonsson 2008.

22. Stephan S, Hippler J, Köhler T, Deep A, Schmidt T, Schmitz OJ Contaminant screening of wastewater with HPLC-IM-qTOF-MS and LC+LCIM-qTOF-MS using a CCS database. Anal Bioanal Chem. 2016;408:6545-55.

23. Duff RB, Bacon JS, Mundie CM, Farmer VC, Russell JD, Forrester AR. Catalpol and methylcatalpol: naturally occurring glycosides in Plantago and buddleia species. Biochem J. 1965;96:1-5.

24. Endo K, Takahashi K, Abe T, Hikino H. Structure of forsythoside$\mathrm{B}$, an antibacterial principle of Forsythia-Koreana stems. J Heterocyclic Chem. 1982;19:261-4.

25. Gross G-A, Sticher O, Calis I. Phenylpropanoid glycosides isolated from Scrophularia scopolii. Phytochemistry. 1987;26:2057-61.

26. Kitagawa S, Tsukamoto H, Hisada S, Nishibe S. Studies on the chinese crude drug "forsythiae fructus."vii. A new caffeoyl glycoside from forsythia viridissima. Chem Pharm Bull. 1984;32:1209-13.

27. Matsuda H, Cai H, Kubo M, Tosa H, Iinuma M. Study on anticataract drugs from natural sources. II. Effects of buddlejae flos on in vitro aldose reductase activity. Biol Pharm Bull. 1995;18:463-6.

28. Pfander H, Wittwer F. Carotinoid-glycoside. 3. Mitteilung. untersuchungen zur carotinoidzusammensetzung im safran. Helv Chim Acta. 1975;58:2233-6.

29. Zimin L, Zhongjian J. Phenylpropanoid and iridoid glycosides from Pedicularis striata. Phytochemistry. 1991;30:1341-4.

Publisher's note Springer Nature remains neutral with regard to jurisdictional claims in published maps and institutional affiliations.

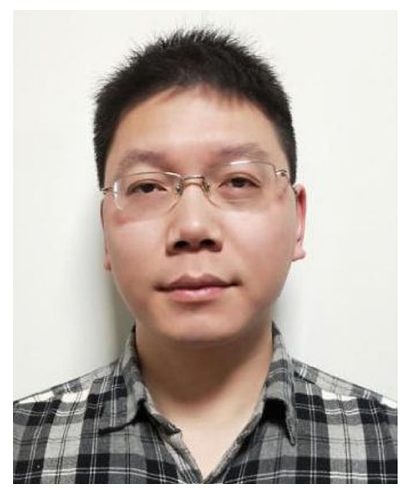

Yingzhuang Chen is Head of the Two-Dimensional Liquid Chromatography Team at the Ke y L a b or a tory of Phytochemical R\&D of Hunan Province and Key Laboratory of Chemical Biology \& Traditional Chinese Medicine Research, Ministry of Education in China, and he has been working for several years on the development of monolithic stationary phase, high orthogonal two-dimensional liquid chromatography, and other new analytical techniques for deep profiling of complex samples such as Traditional Chinese Medicine (TCM). From May 2018 to May 2019, he studied in Prof. Oliver's group as a visiting scholar. 


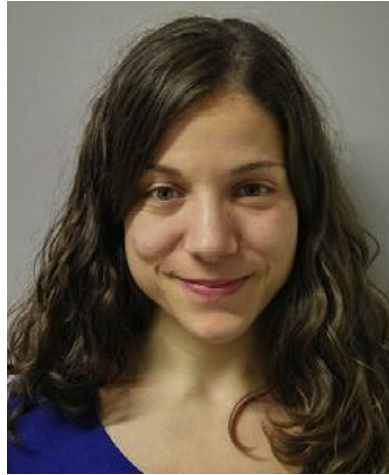

Lidia Montero is a postdoctoral researcher in the Applied Analytical Chemistry Department of the University of Duisburg-Essen in Germany where she is working on the application of 2DLC methods and other multidimensional separations for the analysis of a great variety of samples. She obtained her Ph.D in 2017 from the Food Science Research Institute (CIAL) belonging to the Spanish National Research Council. The objective of her thesis was the development of LCxLC methods for the analysis of potential bioactive secondary metabolites of fruits and food-related plants.

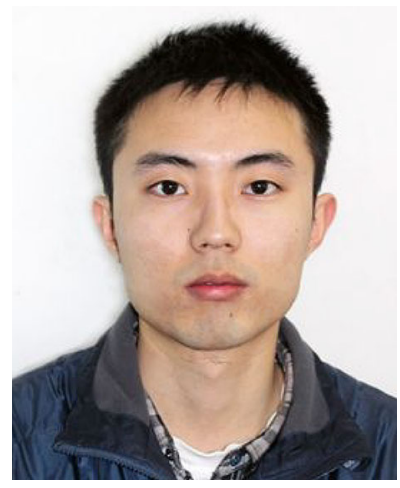

Jiang Luo grew up in the north of China. In September 2015, he started his study at the University of Duisburg-Essen, where he completed his bachelor degree in the group of Prof. Oliver. Schmitz in 2019. After that, he has continued his master's study there.

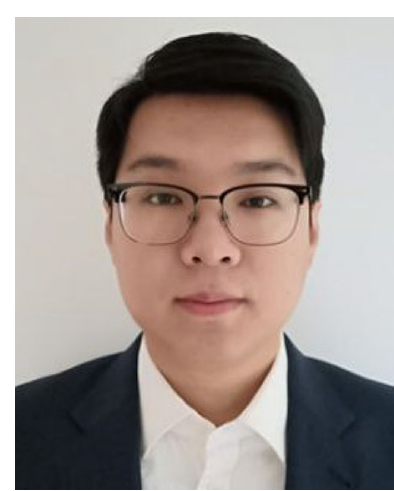

chromatography.

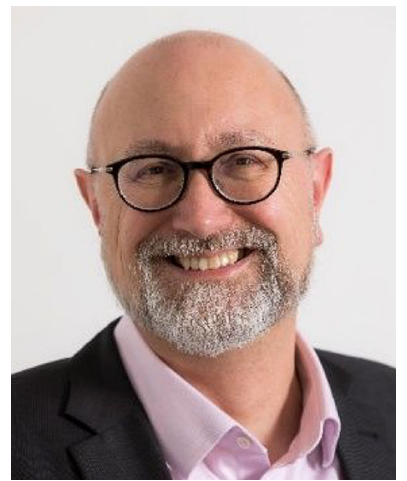

Junjie $\mathbf{L i}$ is a $\mathrm{PhD}$ student from the group of Prof. Oliver J. Schmitz at the University of Duisburg-Essen. He has been working on two-dimensional gas chromatography for several years on bacterial metabolites (such as Cobetia Marina and Sulfolobus Acidocaldarius) profiling under stress (ozone, starving, etc.) based on mass spectrometry as well as cooperating together with Dr. Yingzhuang Chen for the development of the at-column modulator on two-dimensional liquid

Oliver J. Schmitz is Head of Applied Analytical Chemistry and the Teaching and Research Center for Separation at the University of Duisburg-Essen. $\mathrm{He}$ is co-founder of iGenTraX UG and member of several editorial boards. His research topics are the development of new ion sources, multidimensional chromatography, metabolomics, and origin-of-life. 